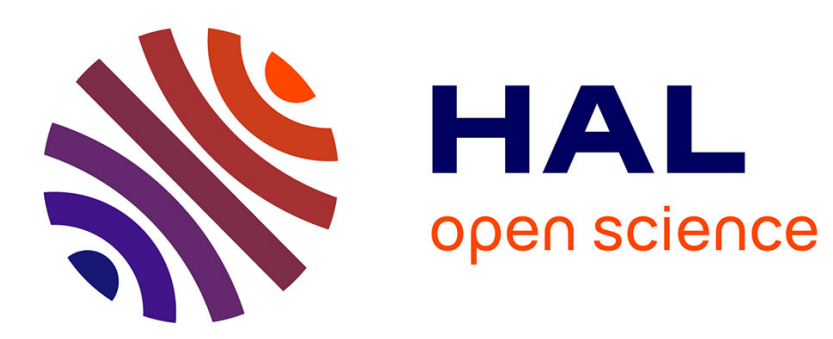

\title{
Grounding Awareness on Belief Bases
}

\author{
Emiliano Lorini, Pengfei Song
}

\section{To cite this version:}

Emiliano Lorini, Pengfei Song. Grounding Awareness on Belief Bases. Third International Workshop on Dynamic Logic: New Trends and Applications (DALI 2020), Nov 2020, Prague, Czech Republic. pp.170-186, 10.1007/978-3-030-65840-3_11. hal-03008597

\section{HAL Id: hal-03008597 https://hal.science/hal-03008597}

Submitted on 13 Dec 2020

HAL is a multi-disciplinary open access archive for the deposit and dissemination of scientific research documents, whether they are published or not. The documents may come from teaching and research institutions in France or abroad, or from public or private research centers.
L'archive ouverte pluridisciplinaire HAL, est destinée au dépôt et à la diffusion de documents scientifiques de niveau recherche, publiés ou non, émanant des établissements d'enseignement et de recherche français ou étrangers, des laboratoires publics ou privés. 


\title{
Grounding Awareness on Belief Bases
}

\author{
Emiliano Lorini ${ }^{1}[$ https://orcid.org/0000-0002-7014-6756] and \\ Pengfei Song2,1[https://orcid.org/0000-0002-6271-9307] \\ 1 IRIT-CNRS, Toulouse University, France \\ 2 Department of Philosophy, Institute of Logic and Cognition, \\ Sun Yat-Sen University, Guangzhou, China
}

\begin{abstract}
We introduce a multi-agent logic of explicit, implicit belief and awareness with a semantics using belief bases. The novelty of our approach is that an agent's awareness is not a primitive but is directly computed from the agent's belief base. We prove soundness and completeness of the logic relative to the belief base semantics. Furthermore, we provide a polynomial embedding of the logic of propositional awareness into it.
\end{abstract}

\section{Introduction}

The notion of awareness was introduced in the area of epistemic logic by Fagin \& Halpern (F\&H) [4] to cope with the problem of logical omniscience [10]. Their approach is syntactic to the extent that they associate a subset of formulas to each agent at each state, indicating the formulas the agent is aware of. Following the idea suggested by Levesque [11], F\&H make the distinction between explicit belief and implicit belief, where explicit belief is defined to be implicit belief plus awareness.

There is another tradition in the formalization of awareness, initiated by Modica \& Rustichini $[15,16]$ and Heifetz et al. $[8,9]$. They support a semantic approach by letting possible worlds be associated with a subset of all propositional variables being defined. Hence, an agent is aware of a formula if and only if, every atomic proposition occurring in the formula is defined at every epistemically accessible state for the agent. Such a notion of awareness is often called propositional awareness in opposition to the notion of general awareness, according to which an agent can be "primitively" aware not only of atomic propositions but also of complex formulas. Halpern [5] proves an equivalence result between the syntactic approach and the semantic approach to propositional awareness in a single-agent setting. Moreover, Halpern \& Rêgo [6] present an analogous equivalence result for multi-agent awareness structures. van Ditmarsch et al. [3] give a novel notion called speculative knowledge, which is also built on propositional awareness.

The concept of explicit belief, which is central in the logic of awareness, is closely related to the concept of belief base $[17,14,7,18]$. The latter plays an important role in the AGM approach to belief revision [1] and, more generally, in the area of knowledge representation and reasoning (KR). Recently, in $[12,13]$ we 
defined a formal semantics for multi-agent epistemic logic exploiting belief bases which clearly distinguishes explicit from implicit belief. Specifically, according to this semantics, an agent explicitly believes that a certain fact $\alpha$ is true if $\alpha$ is a piece of information included in the agent's belief base. On the contrary, the agent implicitly believes that $\alpha$, if $\alpha$ is derivable from the agent's belief base. A logic of explicit and implicit belief, called Logic of Doxastic Attitudes (LDA), was defined on the top of this semantics.

In this paper, we extend the semantics introduced in [12] and the corresponding logic LDA with propositional awareness. We call LDAA the resulting logic. The novelty of our approach lies in the fact that the notion of awareness is not primitive but is directly computed from the notion of belief base. In particular, for an agent to be aware of a proposition $p, p$ has to be included in the agent's vocabulary, that is to say, there should exist a formula in the agent's belief base which contains $p$. From this perspective, we offer a minimalistic logic approach to explicit, implicit belief and awareness in which only the former concept is primitive, while the other two concepts are defined from it.

The paper is organized as follows. In Section 2, we present the language of our logic of explicit, implicit belief and awareness. In Section 3, we first present the belief base semantics with respect to which the language is interpreted. Then, we introduce two alternative semantics which are closer in spirit to the standard semantics for epistemic logic based on multi-relational Kripke structures. We show that the three semantics are all equivalent with respect to the language under consideration. Section 4 is devoted to axiomatic results for our logic, while in Section 5 we explore the connection between our logic and Halpern's logic of propositional awareness (LPA) [5], by providing a satisfiability-preserving embedding of the latter into the former. Finally, in Section 6 we conclude.

\section{Language}

This section presents the language of the Logic of Doxastic Attitudes with Awareness (LDAA) to represent explicit beliefs, implicit beliefs, and awareness. It extends the language in [12] by the awareness modality. Let $\operatorname{Atm}=\{p, q, \ldots\}$ be a countably infinite set of atomic propositions and let $A g t=\{1, \ldots, n\}$ be a finite set of agents. The language is given by the two levels in the following definition.

Definition 1. The language $\mathcal{L}_{0}($ Atm, Agt $)$ is defined as follows:

$$
\alpha::=p|\neg \alpha| \alpha_{1} \wedge \alpha_{2}\left|\triangle_{i} \alpha\right| \bigcirc_{i} \alpha
$$

where $p$ ranges over Atm and $i$ ranges over Agt. The language $\mathcal{L}_{\mathrm{LDAA}}($ Atm, Agt $)$ extends $\mathcal{L}_{0}($ Atm, Agt $)$ by implicit belief operators and is defined as follows:

$$
\varphi::=\alpha|\neg \varphi| \varphi_{1} \wedge \varphi_{2}\left|\square_{i} \varphi\right| \bigcirc_{i} \varphi
$$

where $i$ ranges over Agt. 
When it is unambiguous from the context, we write $\mathcal{L}_{0}$ instead of $\mathcal{L}_{0}(A t m, A g t)$ and $\mathcal{L}_{\text {LDAA }}$ instead of $\mathcal{L}_{\text {LDAA }}($ Atm, Agt $)$. The other Boolean connectives $\vee, \rightarrow$, $\leftrightarrow, \top$ and $\perp$ are defined from $\neg$ and $\wedge$ in the standard way. The formula $\triangle_{i} \alpha$ is read "agent $i$ explicitly believes that $\alpha$ is true". The formula $\bigcirc_{i} \alpha$ is read "agent $i$ is aware of $\alpha$ ". The $\triangle_{i}$-operator can be iterated, which means that the language contains expressions for higher-order explicit beliefs, such as $\triangle_{i} \triangle_{j} \alpha$, which is read "agent $i$ explicitly believes that agent $j$ explicitly believes that $\alpha$ is true". The iteration is possibly a mix of explicit belief and awareness, such as $\triangle_{i} \bigcirc_{j} \alpha$, which is read "agent $i$ explicitly believes that agent $j$ is aware of $\alpha$ ".

And the formula $\square_{i} \varphi$ is read "agent $i$ implicitly believes that $\varphi$ is true". The dual operator $\diamond_{i}$ is defined as follows:

$$
\diamond_{i} \varphi:=\neg \square_{i} \neg \varphi
$$

where $\diamond_{i} \varphi$ is read " $\varphi$ is consistent with agent $i$ 's explicit beliefs".

Note that the modality $\bigcirc_{i}$ appears at both levels of the language, but the modality $\triangle_{i}$ only appears at the first level. As a result, we can have awareness operators in the scope of explicit belief operators, but not implicit belief operators. Moreover, both explicit belief operator and implicit belief operator are allowed inside the awareness operator. It is for the reason that, the concept of propositional awareness allows awareness of any formula that is constituted by atomic propositions that the agent is aware of.

Since we represent a propositional notion of awareness, i.e., being aware of a formula is equivalent to being aware of every atomic proposition occurring in it, we need the following inductive definition to represent the set of atomic propositions occurring in a formula $\varphi$, denoted by $\operatorname{Atm}(\varphi)$ :

$-\operatorname{Atm}(p):=\{p\}$,

$-\operatorname{Atm}(\neg \varphi):=\operatorname{Atm}(\varphi)$,

$-\operatorname{Atm}\left(\varphi_{1} \wedge \varphi_{2}\right):=\operatorname{Atm}\left(\varphi_{1}\right) \cup \operatorname{Atm}\left(\varphi_{2}\right)$,

- $\operatorname{Atm}\left(X_{i} \varphi\right):=\operatorname{Atm}(\varphi)$, for $X \in\{\triangle, \bigcirc, \square\}$.

Let $Y \subseteq \mathcal{L}_{\text {LDAA }}$ be finite, we define $\operatorname{Atm}(Y):=\bigcup_{\varphi \in Y} \operatorname{Atm}(\varphi)$.

\section{Semantics}

In this section, we present three families of formal semantics for $\mathcal{L}_{\text {LDAA }}$. The first semantics exploits belief bases. An agent's set of doxastic alternatives and awareness set are not primitive but computed from them. The second semantics is a Kripke-style semantics, in which we require each agent's set of doxastic alternatives to be equal to the set of worlds in which his explicit beliefs are true, and the agent's awareness set to be equal to the set of of atomic propositions occurring in his explicit beliefs. The third semantics relaxes these requirements, so that an agent's set of doxastic alternatives is included in the set of worlds in which the agent's explicit beliefs are true, and the set of atomic propositions occurring in an agent's explicit beliefs is a subset of the agent's awareness set. 


\subsection{Multi-agent belief-awareness base semantics}

Let us start with the definition of belief-awareness base.

Definition 2. A multi-agent belief-awareness base is a tuple $B A=\left(B_{1}, \ldots, B_{n}\right.$, $\left.A_{1}, \ldots, A_{n}, V\right)$ where,

- $B_{i} \subseteq \mathcal{L}_{0}$ is agent $i$ 's belief base for any $i \in A g t$,

- $A_{i}=\operatorname{Atm}\left(B_{i}\right)$ is agent $i$ 's awareness set for any $i \in A g t$,

$-V \subseteq$ Atm is the actual state.

The set of all multi-agent belief-awareness bases is denoted by BA. With the definition of multi-agent belief-awareness bases, we have the following interpretations for $\mathcal{L}_{0}$.

Definition 3. For any $B A=\left(B_{1}, \ldots, B_{n}, A_{1}, \ldots, A_{n}, V\right) \in \mathbf{B A}$ :

- $B A \models p$ iff $p \in V$,

$-B A \models \neg \alpha$ iff $B A \not \models \alpha$,

$-B A=\alpha_{1} \wedge \alpha_{2}$ iff $B A=\alpha_{1}$ and $B A=\alpha_{2}$,

$-B A=\triangle_{i} \alpha$ iff $\alpha \in B_{i}$,

- $B A \models \bigcirc_{i} \alpha$ iff $\operatorname{Atm}(\alpha) \subseteq A_{i} \cdot{ }^{3}$

By the interpretation, our awareness is propositional, i.e., being aware of a formula is equivalent to being aware of every atomic proposition occurring in the formula. Such a notion of awareness is different with the notion of general awareness according to which an agent can be aware of $p \wedge q$ without being aware of $p \vee q$.

The following definition introduces the concept of multi-agent belief-awareness model.

Definition 4. A multi-agent belief-awareness model (MABA) is a pair $(B A, C x t)$, where $B A \in \mathbf{B A}$ and $C x t \subseteq \mathbf{B A}$.

$C x t$ is the agents' context or common ground [19]. It corresponds to the body of information that the agents share and that they use to make inferences from their explicit beliefs. Following [12], in the following definition we compute the agents' doxastically accessibility relations from their belief bases.

Definition 5. For any $i \in A g t, \mathcal{R}_{i}$ is the binary relation on $\mathbf{B A}$ such that for any $B A=\left(B_{1}, \ldots, B_{n}, A_{1}, \ldots, A_{n}, V\right), B A^{\prime}=\left(B_{1}^{\prime}, \ldots, B_{n}^{\prime}, A_{1}^{\prime}, \ldots, A_{n}^{\prime}, V^{\prime}\right) \in \mathbf{B A}$,

$\left(B A, B A^{\prime}\right) \in \mathcal{R}_{i}$ if and only if $\forall \alpha \in B_{i}, B A^{\prime}=\alpha$.

With the accessibility relation defined, we have the following definition of interpretations for formulas in $\mathcal{L}_{\text {LDAA }}$. The boolean case is defined in the usual way and omitted.

\footnotetext{
${ }^{3}$ Note that the awareness component of Definition 2 seems unnecessary, as we could interpret it equivalenty by postulating " $B A \models \bigcirc_{i} \alpha$ iff $\operatorname{Atm}(\alpha) \subseteq \operatorname{Atm}\left(B_{i}\right)$ ". We keep it for the reason that it has counterparts in NDAM semantics and quasi-NDAM semantics hereinafter. In quasi-NDAM semantics, an agent's awareness set is supposed to be a superset of the set of atomic propositions occurring in the agent's belief set.
} 
Definition 6. Let $(B A, C x t)$ be a MABA with $B A=\left(B_{1}, \ldots, B_{n}, A_{1}, \ldots, A_{n}, V\right)$. Then,

- $(B A, C x t) \models \alpha$ iff $B A \models \alpha$,

- $(B A, C x t) \models \square_{i} \varphi$ iff $\forall B A^{\prime} \in C x t$, if $\left(B A, B A^{\prime}\right) \in \mathcal{R}_{i}$ then $\left(B A^{\prime}, C x t\right) \models \varphi$,

- $(B A, C x t) \models \bigcirc_{i} \varphi$ iff $\operatorname{Atm}(\varphi) \subseteq A_{i}$.

The following two definitions specify two interesting properties of MABAs.

Definition 7. The MABA $(B A, C x t)$ satisfies global consistency $(G C)$ if and only if, for any $i \in A g t$ and for any $B A^{\prime} \in(\{B A\} \cup C x t)$, there exists $B A^{\prime \prime} \in C x t$ such that $\left(B A^{\prime}, B A^{\prime \prime}\right) \in \mathcal{R}_{i}$.

Definition 8. The MABA $(B A, C x t)$ satisfies belief correctness $(B C)$ if and only if $B A \in C x t$ and, for any $i \in A g t$ and for any $B A^{\prime} \in C x t,\left(B A^{\prime}, B A^{\prime}\right) \in \mathcal{R}_{i}$.

For $\mathrm{X} \subseteq\{\mathrm{GC}, \mathrm{BC}\}, \mathbf{M A B A}_{\mathrm{X}}$ is the class of MABAs satisfying all the conditions in X. MABA $\mathbf{A}_{\emptyset}$ is the class of all MABAs, and we write MABA instead of $\mathbf{M A B A} \mathbf{A}_{\emptyset}$. It is easy to see that $\mathbf{M A B} \mathbf{A}_{\{\mathrm{GC}, \mathrm{BC}\}}=\mathbf{M A B A}_{\{\mathrm{BC}\}}$.

Let $\varphi \in \mathcal{L}_{\text {LDAA, }}$ we say that $\varphi$ is valid for the class $\mathbf{M A B} \mathbf{A}_{\mathrm{X}}$ if and only if, for every $(B A, C x t) \in \mathbf{M A B A}_{\mathbf{X}}$ we have $(B A, C x t) \models \varphi$. We say that $\varphi$ is satisfiable of the class $\mathbf{M A B} \mathbf{A}_{\mathrm{X}}$ if and only if $\neg \varphi$ is not valid for the class MABA $_{X}$

\subsection{Notional Model Semantics}

In this section we introduce an alternative Kripke-style semantics for the language $\mathcal{L}_{\text {LDAA }}$ based on notional doxastic-awareness model which extend notional doxastic models defined in $[12,13]$ by awareness functions.

Definition 9. A notional doxastic-awareness model (NDAM) is a tuple $\mathcal{M}=$ $(\mathrm{W}, \mathrm{D}, \mathrm{A}, \mathrm{N}, \mathrm{V})$ where,

- W is a non-empty set of worlds,

- D: Agt $\times W \longrightarrow 2^{\mathcal{L}_{0}}$ is a doxastic function,

- A: Agt $\times W \longrightarrow 2^{\text {Atm }}$ is an awareness function,

$-\mathrm{N}:$ Agt $\times W \longrightarrow 2^{\mathrm{W}}$ is a notional function,

$-\mathrm{V}:$ Atm $\longrightarrow 2^{\mathrm{W}}$ is a valuation function.

and such that, given the following inductive definition of the semantic interpretation of formulas in $\mathcal{L}_{\mathrm{LDAA}}$ :

$-(\mathcal{M}, w) \models p$ iff $w \in \mathrm{V}(p)$,

$-(\mathcal{M}, w) \models \neg \varphi$ iff $(\mathcal{M}, w) \not \models \varphi$,

- $(\mathcal{M}, w)=\varphi \wedge \psi$ iff $(\mathcal{M}, w) \models \varphi$ and $(\mathcal{M}, w) \models \psi$,

$-(\mathcal{M}, w)=\triangle_{i} \alpha$ iff $\alpha \in \mathrm{D}(i, w)$,

- $(\mathcal{M}, w) \models \square_{i} \varphi$ iff $\forall u \in \mathrm{N}(i, w),(\mathcal{M}, u) \models \varphi$,

- $(\mathcal{M}, w) \models \bigcirc_{i} \varphi$ iff $\operatorname{Atm}(\varphi) \subseteq \mathrm{A}(i, w)$. 
it satisfies the following conditions (C1) and (C2), for all $i \in$ Agt and for all $w \in \mathrm{W}:$

(C1) $\mathrm{A}(i, w)=\operatorname{Atm}(\mathrm{D}(i, w))$

(C2) $\mathrm{N}(i, w)=\bigcap_{\alpha \in \mathrm{D}(i, w)}\|\alpha\|_{M}$, where $\|\alpha\|_{M}=\{u \in \mathrm{W} \mid(\mathcal{M}, u) \models \alpha\}$.

The following definitions specify global consistency (GC) and belief correctness (BC) for notional models.

Definition 10. The NDAM $\mathcal{M}=(\mathrm{W}, \mathrm{D}, \mathrm{A}, \mathrm{N}, \mathrm{V})$ satisfies global consistency if and only if, for any $i \in$ Agt and for any $w \in \mathrm{W}, \mathrm{N}(i, w) \neq \emptyset$.

Definition 11. The NDAM $\mathcal{M}=(\mathrm{W}, \mathrm{D}, \mathrm{A}, \mathrm{N}, \mathrm{V})$ satisfies belief correctness if and only if, for any $i \in$ Agt and for any $w \in \mathrm{W}, w \in \mathrm{N}(i, w)$.

For any $\mathrm{X} \subseteq\{\mathrm{GC}, \mathrm{BC}\}, \mathbf{N D A M}_{\mathrm{X}}$ is the class of NDAMs satisfying the conditions in X. NDAM $\mathbf{N}_{\emptyset}$ is the class of all NDAMs, and we write NDAM instead of $\mathbf{N D A M}_{\emptyset}$. Analogously to MABAs, we have $\mathbf{N D A M}_{\{\mathrm{GC}, \mathrm{BC}\}}=\mathbf{N D A M}_{\{\mathrm{BC}\}}$. A NDAM $\mathcal{M}=(\mathrm{W}, \mathrm{D}, \mathrm{A}, \mathrm{N}, \mathrm{V})$ is finite if and only if $\mathrm{W}, \mathrm{D}(i, w)$, and $\mathrm{V}^{\leftarrow}(w)$ are finite sets for any $i \in A g t$ and any $w \in \mathrm{W}$, where $\mathrm{V}^{\leftarrow}(w)=\{p \in \operatorname{Atm} \mid w \in \mathrm{V}(p)\}$. As $A(i, w)=\operatorname{Atm}(\mathrm{D}(i, w))$, it follows that, if a NDAM $\mathcal{M}$ is finite, $A(i, w)$ is also a finite set for any $i \in A g t$ and any $w \in \mathrm{W}$. We use finite-NDAM $\mathbf{X}_{\mathbf{X}}$ to denote the class of finite NDAMs satisfying the conditions in $X$.

Let $\varphi \in \mathcal{L}_{\text {LDAA }}$, we say that $\varphi$ is valid for the class $\operatorname{NDAM}_{\mathrm{X}}$ if and only if, for every $\mathcal{M}=(\mathrm{W}, \mathrm{D}, \mathrm{A}, \mathrm{N}, \mathrm{V}) \in \mathbf{N D A M}_{\mathrm{X}}$ and for every $w \in \mathrm{W}$, we have $(\mathcal{M}, w) \models \varphi$. We say that $\varphi$ is satisfiable for the class $\mathbf{N D A M}_{\mathbf{X}}$ if and only if $\neg \varphi$ is not valid for the class NDAM $_{\mathrm{X}}$.

\subsection{Quasi-Model Semantics}

This section provides an alternative semantics for the language $\mathcal{L}_{\text {LDAA }}$ based on a more general class of models, called quasi-notional doxastic-awareness models (quasi-NDAMs) in which the restrictions on the notional and awareness function are weakened.

Definition 12. A quasi-notional doxastic-awareness model (quasi-NDAM) is a tuple $\mathcal{M}=(\mathrm{W}, \mathrm{D}, \mathrm{A}, \mathrm{N}, \mathrm{V})$ where $\mathrm{W}, \mathrm{D}, \mathrm{A}, \mathrm{N}$ and $\mathrm{V}$ are as in Definition 9 except that Condition $C 1$ and $C 2$ are replaced by the following weaker conditions, for all $i \in$ Agt and for all $w \in \mathrm{W}$ :

$\left(C 1^{*}\right) \mathrm{A}(i, w) \supseteq \operatorname{Atm}(\mathrm{D}(i, w))$,

$\left(C 2^{*}\right) \mathrm{N}(i, w) \subseteq \bigcap_{\alpha \in \mathrm{D}(i, w)}\|\alpha\|_{M}$.

As for NDAMs, for any $\mathrm{X} \subseteq\{\mathrm{GC}, \mathrm{BC}\}, \mathrm{QNDAM}_{\mathrm{X}}$ is the class of quasiNDAMs satisfying the conditions in X. QNDAM $\mathbf{Q}_{\emptyset}$ is the class of all quasiNDAMs, and we write QNDAM instead of QNDAM $\mathbf{Q}_{\emptyset}$. As for MABAs and NDAMs, we have QNDAM $_{\{\mathrm{GC}, \mathrm{BC}\}}=\mathbf{Q N D A M}_{\{\mathrm{BC}\}}$. A quasi-NDAM $\mathcal{M}=$ $(\mathrm{W}, \mathrm{D}, \mathrm{A}, \mathrm{N}, \mathrm{V})$ is finite if $\mathrm{W}, \mathrm{D}(i, w), \mathrm{A}(i, w)$ and $\mathrm{V}^{\leftarrow}(w)$ are finite sets for any $i \in$ Agt and any $w \in \mathrm{W}$. We use finite-QNDAM $\mathbf{M}_{\mathbf{X}}$ to denote the class of finite quasi-NDAMs satisfying the conditions in $X$. Validity and satisfiability of formulas for a class $\mathbf{Q N D A M}_{\mathrm{X}}$ are defined in the usual way. 


\subsection{Equivalence Results}

In this section, we present equivalence results between the five different semantics for $\mathcal{L}_{\text {LDAA }}$ we presented above (i.e., MABA, NDAM, finite-NDAM, QNDAM, and finite-QNDAM).

\section{Equivalence between quasi-NDAMs and finite quasi-NDAMs}

First of all, we consider the relationship between QNDAM and finite-QNDAM. Let us define a filtrated model for the proof.

Let $\mathcal{M}=(\mathrm{W}, \mathrm{D}, \mathrm{A}, \mathrm{N}, \mathrm{V})$ be a (possibly infinite) quasi-NDAM and let $\Sigma \subseteq$ $\mathcal{L}_{\text {LDAA }}$ be an arbitrary finite set of formulas which is closed under subformulas. The equivalence relation $\equiv_{\Sigma}$ on $\mathrm{W}$ is defined as follows:

$$
\equiv_{\Sigma}=\{(w, v) \in \mathrm{W} \times \mathrm{W}: \forall \varphi \in \Sigma,(\mathcal{M}, w) \models \varphi \text { iff }(\mathcal{M}, v) \models \varphi\} .
$$

Let $[w]_{\Sigma}$ be the equivalence class of the world $w$ generated by the relation $\equiv_{\Sigma}$. The model $\mathcal{M}_{\Sigma}=\left(\mathrm{W}_{\Sigma}, \mathrm{D}_{\Sigma}, \mathrm{A}_{\Sigma}, \mathrm{N}_{\Sigma}, \mathrm{V}_{\Sigma}\right)$ is the filtration of $\mathcal{M}$ under $\Sigma$ where,

$-\mathrm{W}_{\Sigma}=\left\{[w]_{\Sigma} \mid w \in \mathrm{W}\right\}$

- for any $i \in A g t$ and for any $[w]_{\Sigma} \in \mathrm{W}_{\Sigma}, \mathrm{D}_{\Sigma}\left(i,[w]_{\Sigma}\right)=\left(\bigcap_{w \in[w]_{\Sigma}} \mathrm{D}(i, w)\right) \cap \Sigma$,

- for any $i \in A g t$ and for any $[w]_{\Sigma} \in \mathrm{W}_{\Sigma}, \mathrm{A}_{\Sigma}\left(i,[w]_{\Sigma}\right)=\left(\bigcap_{w \in[w]_{\Sigma}} \mathrm{A}(i, w)\right) \cap \Sigma$,

- for any $i \in A g t$ and for any $[w]_{\Sigma} \in \mathrm{W}_{\Sigma}, \mathrm{N}_{\Sigma}\left(i,[w]_{\Sigma}\right)=\left\{[u]_{\Sigma} \in \mathrm{W}_{\Sigma} \mid \exists w \in\right.$ $[w]_{\Sigma}, \exists u \in[u]_{\Sigma}$ such that $\left.u \in \mathrm{N}(i, w)\right\}$,

- for any $p \in \operatorname{Atm}, \mathrm{V}_{\Sigma}(p)=\left\{[w]_{\Sigma} \mid(\mathcal{M}, w) \models p\right\}$ if $p \in \operatorname{Atm}(\Sigma), \mathbb{V}_{\Sigma}(p)=\emptyset$ otherwise.

We have the following filtration lemma showing that the filtrated model is semantically equivalent with the original model with respect to $\Sigma$.

Lemma 1. Let $\varphi \in \Sigma$ and let $w \in \mathrm{W}$. Then, $(\mathcal{M}, w) \models \varphi$ if and only if $\left(\mathcal{M}_{\Sigma},[w]_{\Sigma}\right) \models \varphi$.

Proof. The proof is by induction on the structure of $\varphi$. For the cases other than $\varphi=\bigcirc_{i} \psi$, the proof is identical with that of Lemma 4 in the appendix of [12]. So we only need to prove the case when $\varphi=\bigcirc_{i} \psi$.

$(\Rightarrow)$ Suppose $(\mathcal{M}, w) \models \bigcirc_{i} \psi$ with $\bigcirc_{i} \psi \in \Sigma$. Thus, $\operatorname{Atm}(\psi) \subseteq \mathrm{A}(i, w)$. Hence, by the definition of $\mathrm{A}_{\Sigma}\left(i,[w]_{\Sigma}\right)$ and the fact that $\Sigma$ is closed under subformulas, we have $\operatorname{Atm}(\psi) \subseteq \mathrm{A}_{\Sigma}\left(i,[w]_{\Sigma}\right)$. It follows that $\left(\mathcal{M}_{\Sigma},[w]_{\Sigma}\right) \models \bigcirc_{i} \psi$.

$(\Leftarrow)$ For the other direction, suppose $\left(\mathcal{M}_{\Sigma},[w]_{\Sigma}\right) \models \bigcirc_{i} \psi$ with $\bigcirc_{i} \psi \in \Sigma$. Thus, $\operatorname{Atm}(\psi) \subseteq \mathrm{A}_{\Sigma}\left(i,[w]_{\Sigma}\right)$. Hence, by the definition of $\mathrm{A}_{\Sigma}\left(i,[w]_{\Sigma}\right), \operatorname{Atm}(\psi) \subseteq$ $\mathrm{A}(i, w)$.

The following proposition highlights that $\mathcal{M}_{\Sigma}$ is finite and preserves the properties of $\mathcal{M}$. 
Proposition 1. $\mathcal{M}_{\Sigma}=\left(\mathrm{W}_{\Sigma}, \mathrm{D}_{\Sigma}, \mathrm{A}_{\Sigma}, \mathrm{N}_{\Sigma}, \mathrm{V}_{\Sigma}\right)$ is a finite quasi-NDAM. Moreover, for any $\mathrm{X} \in\{\mathrm{GC}, \mathrm{BC}\}$, if $\mathcal{M}$ satisfies $\mathrm{X}$, then $\mathcal{M}_{\Sigma}$ also satisfies it.

Proof. By the proof of Proposition 12 in the appendix of [12], we have that, $\mathcal{M}_{\Sigma}$ is finite and satisfies Condition $\left(\mathrm{C} 2^{*}\right)$ in Definition 12 , and that, for any $\mathrm{X} \in$ $\{\mathrm{GC}, \mathrm{BC}\}$, if $\mathcal{M}$ satisfies X, then $\mathcal{M}_{\Sigma}$ also satisfies it. Here, we only need to prove that $\mathcal{M}$ satisfies Condition $\left(\mathrm{C} 1^{*}\right)$ in Definition 12. Suppose $\varphi \in \mathrm{D}_{\Sigma}\left(i,[w]_{\Sigma}\right)$, we need to prove that $\operatorname{Atm}(\varphi) \subseteq \mathrm{A}_{\Sigma}\left(i,[w]_{\Sigma}\right)$. By the definition of $\mathrm{D}_{\Sigma}\left(i,[w]_{\Sigma}\right)$, we have $\varphi \in \mathrm{D}(i, w)$. By Condition $(\mathrm{C} 1 *)$, it follows that, $\operatorname{Atm}(\varphi) \subseteq \mathrm{A}(i, w)$. By the definition of $\mathrm{A}_{\Sigma}\left(i,[w]_{\Sigma}\right)$ and the fact that $\Sigma$ is closed under subformulas, we have $\operatorname{Atm}(\varphi) \subseteq \mathrm{A}_{\Sigma}\left(i,[w]_{\Sigma}\right)$. As a result, $\mathrm{A}_{\Sigma}\left(i,[w]_{\Sigma}\right) \supseteq \operatorname{Atm}\left(\varphi \in \mathrm{D}_{\Sigma}\left(i,[w]_{\Sigma}\right)\right) . \square$

The following lemma is a straighforward consequence of Lemma 1 and Proposition 1.

Lemma 2. Let $\mathrm{X} \in\{\mathrm{GC}, \mathrm{BC}\}$ and $\varphi \in \mathcal{L}_{\mathrm{LDAA}}$. If $\varphi$ is satisfiable for the class $\mathbf{Q N D A M}_{\mathrm{X}}$ then $\varphi$ is satisfiable for the class finite-QNDAM $\mathbf{X}_{\mathbf{X}}$.

\section{Equivalence between finite NDAMs and finite quasi-NDAMs}

Our next result concerns the equivalence between finite-NDAM and finiteQNDAM.

Lemma 3. Let $\mathrm{X} \in\{\mathrm{GC}, \mathrm{BC}\}$ and $\varphi \in \mathcal{L}_{\mathrm{LDAA}}$. If $\varphi$ is satisfiable for the class finite-QNDAM $\mathbf{M}_{\mathrm{X}}$, then $\varphi$ is satisfiable for the class finite-NDAM $\mathbf{M}_{\mathbf{X}}$.

Proof. We are going to build a finite NDAM from a finite quasi-NDAM without changing the satisfiability of $\varphi$. To accomplish this goal, two things are essential in the construction. Firstly, we enlarge each agent's belief base with an identifier proposition to make his set of doxastic alternatives smaller and coincide with his set of notional worlds. Secondly, we combine the identifier with some tautologies by conjunctions, so that the set of atomic propositions occurring in his belief base is equal to his awareness set.

Let $\mathcal{M}=(\mathrm{W}, \mathrm{D}, \mathrm{A}, \mathrm{N}, \mathrm{V})$ be a finite quasi-NDAM that satisfies $\varphi$, i.e., there exists $w \in \mathrm{W}$ such that $(\mathcal{M}, w) \models \varphi$. We define the set of all atomic propositions occurring in some belief base of some agent at some world in $\mathcal{M}$ as follows:

$$
\mathcal{T}(\mathcal{M})=\bigcup_{w \in \mathrm{W}, i \in A g t} \operatorname{Atm}(\mathrm{D}(i, w)) \cup \bigcup_{w \in \mathrm{W}, i \in A g t} \mathrm{~A}(i, w) .
$$

Since $\mathcal{M}$ is finite, $\mathcal{T}(\mathcal{M})$ is also finite.

We have the following injective function which assigns an identifier to each agent and each world in W.

$$
f: A g t \times \mathrm{W} \longrightarrow \operatorname{Atm} \backslash(\mathcal{T}(\mathcal{M}) \cup \operatorname{Atm}(\varphi)) .
$$

As Atm is infinite while $\mathrm{W}, \mathcal{T}(\mathcal{M})$ and $\operatorname{Atm}(\varphi)$ are finite, such an injection exists.

We define a new model $\mathcal{M}^{\prime}=\left(\mathrm{W}^{\prime}, \mathrm{D}^{\prime}, \mathrm{A}^{\prime}, \mathrm{N}^{\prime}, \mathrm{V}^{\prime}\right)$ with $\mathrm{W}^{\prime}=\mathrm{W}, \mathrm{N}^{\prime}=\mathrm{N}$ and where $D^{\prime}, V^{\prime}$ and $A^{\prime}$ are defined as follows: 
- $\mathrm{A}^{\prime}(i, w)=\mathrm{A}(i, w) \cup\{f(i, w)\}$ for every $i \in$ Agt and for every $w \in \mathrm{W}$,

- $\mathrm{D}^{\prime}(i, w)=\mathrm{D}(i, w) \cup\left\{f(i, w) \wedge\left(\bigwedge_{p \in \mathrm{A}(i, w) \backslash \operatorname{Atm}(\mathrm{D}(i, w))}(p \vee \neg p)\right)\right\}$ for every $i \in$ Agt and for every $w \in \mathrm{W}$,

- for every $p \in$ Atm, $\mathrm{V}^{\prime}(p)=\mathrm{V}(p)$ if $p \in \mathcal{T}(\mathcal{M}) \cup \operatorname{Atm}(\varphi)$, $\mathrm{V}^{\prime}(p)=\mathrm{N}(i, w)$ if $p=f(i, w)$,

$\mathrm{V}^{\prime}(p)=\emptyset$ otherwise

It is easy to verify that $\mathcal{M}^{\prime}$ satisfies Condition (C1) and (C2) in Definition 9. Thus, $\mathcal{M}^{\prime}$ is a finite NDAM.

The rest of the proof consists in checking that, for every $\mathrm{X} \in\{\mathrm{GC}, \mathrm{BC}\}$, if $\mathcal{M}$ satisfies $X$ then $\mathcal{M}^{\prime}$ also satisfies $X$, which is straightforward, and that, $(\mathcal{M}, w)=\varphi$ iff $(\mathcal{M}, w) \models \varphi$. We prove the latter by induction on the structure of $\varphi$.

The case $\varphi=p$ is immediate from the definition of $\mathrm{V}^{\prime}$. The boolean cases are straightforward.

Let us prove the case $\varphi=\triangle_{i} \alpha$.

$(\Rightarrow)$ Suppose $(\mathcal{M}, w) \mid=\triangle_{i} \alpha$. Then, we have $\alpha \in \mathrm{D}(i, w)$. Hence, by the definition of $\mathrm{D}^{\prime}, \alpha \in \mathrm{D}^{\prime}(i, w)$. Thus, $\left(\mathcal{M}^{\prime}, w\right) \models \triangle_{i} \alpha$.

$(\Leftarrow)$ Suppose $\left(\mathcal{M}^{\prime}, w\right) \models \triangle_{i} \alpha$. Then, we have $\alpha \in \mathrm{D}^{\prime}(i, w)$. Since $f(i, w) \notin$ $\operatorname{Atm}\left(\triangle_{i} \alpha\right)$, by the definition of $\mathrm{D}^{\prime}$, we have that,

$$
\alpha \neq f(i, w) \wedge\left(\bigwedge_{p \in \mathrm{A}(i, w) \backslash \operatorname{Atm}(\mathrm{D}(i, w))}(p \vee \neg p)\right)
$$

Thus, $\alpha \in \mathrm{D}(i, w)$ and, consequently, $(\mathcal{M}, w) \models \triangle_{i} \alpha$.

Then let us prove the case $\varphi_{i}=\bigcirc_{i} \psi$.

$(\Rightarrow)$ Suppose $(\mathcal{M}, w) \models \bigcirc_{i} \psi$. Then, we have $\operatorname{Atm}(\psi) \subseteq \mathrm{A}(i, w)$. Hence, by the definition of $\mathrm{A}^{\prime}, \operatorname{Atm}(\psi) \subseteq \mathrm{A}^{\prime}(i, w)$. Thus, $\left(\mathcal{M}^{\prime}, w\right) \models \bigcirc_{i} \psi$.

$(\Leftarrow)$ Suppose $\left(\mathcal{M}^{\prime}, w\right) \models \bigcirc_{i} \psi$. Then, we have $\operatorname{Atm}(\psi) \subseteq \mathrm{A}^{\prime}(i, w)$. The definition of $\mathrm{A}^{\prime}$ ensures that $f(i, w) \notin \operatorname{Atm}(\psi)$. Thus, $\operatorname{Atm}(\psi) \subseteq \mathrm{A}(i, w)$ and, consequently, $(\mathcal{M}, w) \models \bigcirc_{i} \psi$.

At last, let us prove the case $\varphi=\square_{i} \psi \cdot(\mathcal{M}, w) \models \square_{i} \psi$ means that $(\mathcal{M}, u) \models \psi$ for all $u \in \mathrm{N}(i, w)$, which is equivalent to $\left(\mathcal{M}^{\prime}, u\right) \models \psi$ for all $u \in \mathrm{N}^{\prime}(i, w)$ by the induction hypothesis and the the fact that $\mathrm{N}^{\prime}(i, w)=\mathbf{N}(i, w)$. The latter means that $\left(\mathcal{M}^{\prime}, w\right) \models \square_{i} \psi$.

Now we have that $(\mathcal{M}, w) \models \varphi$ iff $(\mathcal{M}, w) \models \varphi$. Then, if $\mathcal{M}$ satisfies $\varphi, \mathcal{M}^{\prime}$ satisfies $\varphi$ as well.

\section{Equivalence between MABAs and NDAMs}

The following lemma concerns the equivalence between MABA and NDAM.

Lemma 4. Let $\varphi \in \mathcal{L}_{\mathrm{LDAA}}$ and $\mathrm{X} \in\{\mathrm{GC}, \mathrm{BC}\}$. Then, $\varphi$ is satisfiable for the class $\mathbf{M A B} \mathbf{A}_{\mathbf{X}}$ if and only if $\varphi$ is satisfiable for the class $\mathbf{N D A M}_{\mathbf{X}}$.

Proof. The proof is almost identical to that of Lemma 7 in the appendix of [12]. We leave it to the reader. 


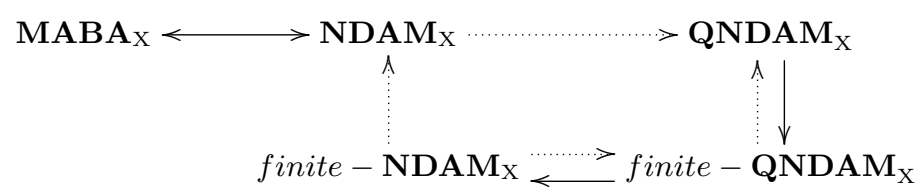

Fig. 1. Relations between semantics for the language $\mathcal{L}_{\text {LDAA }}$. An arrow means that satisfiability relative to the first class of structures implies satisfiability relative to the second class of structures. Full arrows correspond to the results stated in Lemmas 2, 3 and 4 . Dotted arrows denote relations that follow straightforwardly given the inclusion between classes of structures.

Theorem 1. Let $\varphi \in \mathcal{L}_{\mathrm{LDAA}}$ and $\mathrm{X} \subseteq\{\mathrm{GC}, \mathrm{BC}\}$. Then, the following five statements are equivalent:

$-\varphi$ is satisfiable for the class $\mathbf{M A B A _ { X }}$,

$-\varphi$ is satisfiable for the class NDAM $_{\mathbf{X}}$,

$-\varphi$ is satisfiable for the class QNDAM $_{\mathrm{X}}$,

$-\varphi$ is satisfiable for the class finite-QNDAM $\mathbf{M}_{\mathrm{X}}$,

$-\varphi$ is satisfiable for the class finite-NDAM $\mathbf{M}_{\mathbf{X}}$.

Proof. The theorem is a direct consequence of Lemmas 2, 3 and 4.

\section{Axiomatization}

In this section, we define some variants of the LDAA logics and prove their soundness and completeness for their corresponding model classes.

We define the base logic LDAA to be the extension of classical propositional logic given by the following axioms and rule of inference:

$\mathrm{K}_{\square} \cdot\left(\square_{i} \varphi \wedge \square_{i}(\varphi \rightarrow \psi)\right) \rightarrow \square_{i} \psi$

Int $_{\triangle, \square .} \triangle_{i} \alpha \rightarrow \square_{i} \alpha$

Int $\triangle, \bigcirc . \triangle_{i} \alpha \rightarrow \bigcirc_{i} \alpha$

AGPP. $\bigcirc_{i} \varphi \leftrightarrow \bigwedge_{p \in \operatorname{Atm}(\varphi)} \bigcirc_{i} p$

$\mathrm{Nec}_{\square}$. From $\varphi$ infer $\square_{i} \varphi$

For $\mathrm{X} \subseteq\left\{\mathrm{D}_{\square}, \mathrm{T}_{\square}\right\}$, let LDAA $\mathrm{X}$ be the extension of logic LDAA by every axiom in $X$, where:

$D_{\square} \cdot \neg\left(\square_{i} \varphi \wedge \square_{i} \neg \varphi\right)$

$\mathrm{T}_{\square} . \square_{i} \varphi \rightarrow \varphi$

We first prove completeness relative to the quasi-notional model semantics by using a canonical model argument. As usual, we have the following property for maximally consistent sets (MCSs).

Proposition 2. Let $\Gamma$ be a MCS for LDAA . Then:

- if $\varphi, \varphi \rightarrow \psi \in \Gamma$ then $\psi \in \Gamma$, 
$-\varphi \in \Gamma$ or $\neg \varphi \in \Gamma$,

$-\varphi \vee \psi \in \Gamma$ iff $\varphi \in \Gamma$ or $\psi \in \Gamma$.

The following is the Lindenbaum's lemma for our logics. The proof is standard, so we omit it.

Lemma 5. Let $\Gamma$ be a consistent set of formulas for LDAA $_{\mathrm{X}}$, then there exists a MCS $\Gamma^{\prime}$ for LDAA $_{\mathrm{X}}$ such that $\Gamma \subseteq \Gamma^{\prime}$.

To prove completeness with respect to the class $\mathbf{Q N D A M}_{X}$, we construct a canonical model as follows.

Definition 13. Let $\mathrm{X} \subseteq\left\{\mathrm{D}_{\square}, \mathrm{T}_{\square}\right\}$. Then, the canonical model for $\mathrm{LDAA}_{\mathrm{X}}$ is the tuple $\mathcal{M}^{c}=\left(\mathrm{W}^{c}, \mathrm{D}^{c}, \mathrm{~A}^{c}, \mathrm{~N}^{c}, \mathrm{~V}^{c}\right)$ such that:

- $\mathrm{W}^{c}$ is the set of maximally consistent sets (MCSs) for LDAA $\mathrm{X}_{\mathrm{X}}$,

- $\forall w \in \mathrm{W}^{c}, \forall i \in A g t$, and $\forall \alpha \in \mathcal{L}_{0}, \alpha \in \mathrm{D}^{c}(w, i)$ iff $\triangle_{i} \alpha \in w$,

- $\forall w \in \mathrm{W}^{c}, \forall i \in$ Agt, and $\forall p \in A t m, p \in \mathrm{A}^{c}(w, i)$ iff $\bigcirc_{i} p \in w$,

$-\forall w, u \in \mathrm{W}^{c}$ and $\forall i \in$ Agt, $u \in \mathrm{N}^{c}(i, w)$ iff $\forall \varphi \in \mathcal{L}_{\mathrm{LDAA}}$, if $\square_{i} \varphi \in w$ then $\varphi \in u$

- $\forall w \in \mathrm{W}^{c}$ and $\forall p \in$ Atm, $w \in \mathrm{V}^{c}(p)$ iff $p \in w$.

The following existence lemma is necessary for the proof of completeness. We omit its proof since it is completely standard.

Lemma 6. Let $\varphi \in \mathcal{L}_{\mathrm{LDAA}}$ and let $w \in \mathrm{W}^{c}$. Then, if $\diamond_{i} \varphi \in w$ then there exists $u \in \mathrm{N}^{c}(i, w)$ such that $\varphi \in u$.

The following is the truth lemma for our logic.

Lemma 7. Let $\varphi \in \mathcal{L}_{\mathrm{LDAA}}$ and let $w \in \mathrm{W}^{c}$. Then, $\left(\mathcal{M}^{c}, w\right) \models \varphi$ iff $\varphi \in w$.

Proof. The proof is by induction on the structure of the formula $\varphi$. For the cases that $\varphi$ is atomic, Boolean, or of the form $\square_{i} \psi$, the proof is standard by means of Proposition 2 and Lemma 6. The proof for the case $\varphi=\triangle_{i} \alpha$ goes as follows: $\triangle_{i} \alpha \in w$ iff $\alpha \in \mathrm{D}^{c}(i, w)$ iff $\left(\mathcal{M}^{c}, w\right)=\triangle_{i} \alpha$.

For the case $\varphi=\bigcirc_{i} \psi$, by the axiom AGPP, $\bigcirc_{i} \psi \in w$ iff $\forall p \in \operatorname{Atm}(\psi)$, $\bigcirc_{i} p \in w$. By the definition of the canonical model, the latter is equivalent to that, $\forall p \in \operatorname{Atm}(\psi), p \in \mathrm{A}^{c}(w, i)$. The latter is equivalent to $\operatorname{Atm}(\psi) \subseteq \mathrm{A}^{c}(w, i)$, which means $\left(\mathcal{M}^{c}, w\right) \models \bigcirc_{i} \psi$ by our semantics.

We have to show that the canonical model satisfies the corresponding semantic properties if each axiom in $\mathrm{X} \subseteq\left\{\mathrm{D}_{\square}, \mathrm{T}_{\square}\right\}$ is valid in the model. Let us define the following correspondence function between axioms and semantic properties:

$-c f\left(\mathrm{D}_{\square}\right)=\mathrm{GC}$

$-c f\left(\mathrm{~T}_{\square}\right)=\mathrm{BC}$

Proposition 3. Let $\mathrm{X} \subseteq\left\{\mathrm{D}_{\square}, \mathrm{T}_{\square}\right\}$. If $\mathcal{M}^{c}$ is the canonical model for $\mathrm{LDAA}_{\mathrm{X}}$, then it belongs to the class $\mathrm{QNDAM}_{\{c f(x) \mid x \in \mathrm{X}\}}$. 
Proof. Firstly, we need to prove that $\mathcal{M}^{c}$ satisfies Condition $\left(\mathrm{C} 1^{*}\right)$ and $\left(\mathrm{C} 2^{*}\right)$ in Definition 12. For Condition $\left(\mathrm{C} 1^{*}\right)$, we have to prove that if $\alpha \in \mathrm{D}^{c}(i, w)$ then $\operatorname{Atm}(\alpha) \subseteq \mathrm{A}^{c}(i, w)$. Suppose $\alpha \in \mathrm{D}^{c}(i, w)$. Thus, $\triangle_{i} \alpha \in w$. Hence, by the axiom Int $\triangle, \bigcirc, \bigcirc_{i} \alpha \in w$. By the axiom AGPP, it follows that, $\forall p \in \operatorname{Atm}(\alpha), \bigcirc_{i} p \in$ $w$. Then, by the definition of $\mathcal{M}^{c}, \forall p \in \operatorname{Atm}(\alpha), p \in \mathrm{A}^{c}(i, w)$, which means $\operatorname{Atm}(\alpha) \subseteq \mathrm{A}^{c}(i, w)$. For Condition $\left(\mathrm{C} 2^{*}\right)$, we have to prove that if $\alpha \in \mathrm{D}^{c}(i, w)$ then $\mathrm{N}^{c}(i, w) \subseteq\|\alpha\|_{\mathcal{M}^{c}}$. Suppose $\alpha \in \mathrm{D}^{c}(i, w)$. Thus, $\triangle_{i} \alpha \in w$. Hence, by the axiom Int $\triangle, \square, \square_{i} \alpha \in w$. By the definition of $\mathcal{M}^{c}$, if follows that, $\forall u \in \mathrm{N}^{c}(i, w)$, $\alpha \in u$. Thus, by Lemma 7, we have that, $\forall u \in \mathrm{N}^{c}(i, w),\left(\mathcal{M}^{c}, u\right) \models \alpha$. The latter means that $\mathrm{N}^{c}(i, w) \subseteq\|\alpha\|_{\mathcal{M}^{c}}$.

It is easy to verify that $\mathcal{M}^{c}$ has the corresponding properties in $\{c f(x) \mid x \in$ $\mathrm{X}\}$ using the standard proof.

By Lemma 7 and Proposition 3, we are able to prove the following soundness and completeness theorem. Proving soundness is just a routine exercise.

Theorem 2. Let $\mathrm{X} \subseteq\left\{\mathrm{D}_{\square}, \mathrm{T}_{\square}\right\}$. Then, the logic $\mathrm{LDAA}_{\mathrm{X}}$ is sound and complete for the class $\mathbf{Q N D A M} \mathbf{M}_{\{c f(x) \mid x \in \mathrm{X}\}}$.

The following is a corollary of Theorem 1 and Theorem 2.

Corollary 1. Let $\mathrm{X} \subseteq\left\{\mathrm{D}_{\square}, \mathrm{T}_{\square}\right\}$. Then,

- $\operatorname{LDAA}_{\mathrm{X}}$ is sound and complete for the class $\mathbf{N D A M}_{\{c f(x) \mid x \in \mathrm{X}\}}$, and

- $\operatorname{LDAA}_{\mathbf{X}}$ is sound and complete for the class $\mathbf{M A B} \mathbf{A}_{\{c f(x) \mid x \in \mathrm{X}\}}$.

\section{Relationship with Logic of Propositional Awareness}

In this section, we build a connection between LDAA and the logic of propositional awareness (LPA), where the latter, first introduced in [5], is a special case of the logic of general awareness (LGA) by Fagin \& Halpern [4]. Specifically, we provide a polynomial, satisfiability preserving translation of LPA into LDAA. The language of LPA, denoted by $\mathcal{L}_{\mathrm{LPA}}$, is defined by the following grammar:

$$
\varphi::=p|\neg \varphi| \varphi_{1} \wedge \varphi_{2}\left|B_{i} \varphi\right| A_{i} \varphi \mid X_{i} \varphi
$$

where $p$ ranges over Atm and $i$ ranges over Agt. At the semantics level, the logic of propositional awareness exploits awareness structures in which the awareness function is assumed to be propositional.

Definition 14. A propositional awareness model (PAM) is a tuple $\mathcal{M}=(\mathrm{S}, \mathrm{R}, \rho, \pi)$ where:

- $\mathrm{S}$ is a non-empty set of states,

- $\mathrm{R}:$ Agt $\times \mathrm{S} \rightarrow 2^{\mathrm{S}}$ is a doxastic accessibility function,

$-\rho:$ Agt $\times \mathrm{S} \rightarrow 2^{\text {Atm }}$ is a propositional awareness function,

$-\pi:$ Atm $\rightarrow 2^{\mathrm{S}}$ is a valuation function.

The class of propositional awareness models is denoted by PAM. 
We have the following semantic interpretation of formulas in $\mathcal{L}_{\mathrm{LPA}}$ relative to pointed models.

Definition 15. Given a PAM $\mathcal{M}$ and state $s$ in $\mathcal{M}$, formulas in $\mathcal{L}_{\mathrm{LPA}}$ are interpreted relative to $(\mathcal{M}, s)$ as follows:

$-(\mathcal{M}, s) \models p$ iff $s \in \pi(p)$,

$-(\mathcal{M}, s) \models \neg \varphi$ iff $(\mathcal{M}, s) \not \models \varphi$,

- $(\mathcal{M}, s) \models \varphi \wedge \psi$ iff $(\mathcal{M}, s) \models \varphi$ and $(\mathcal{M}, s) \models \psi$,

- $(\mathcal{M}, s) \models B_{i} \varphi$ iff $\forall t \in \mathrm{R}(i, s),(\mathcal{M}, t) \models \varphi$,

- $(\mathcal{M}, s) \models A_{i} \varphi$ iff $\operatorname{Atm}(\varphi) \subseteq \rho(i, s)$,

- $(\mathcal{M}, s) \models X_{i} \varphi$ iff $(\mathcal{M}, s) \models B_{i} \varphi$ and $(\mathcal{M}, s) \models A_{i} \varphi$.

We translate formulas of $\mathcal{L}_{\text {LPA }}$ into formulas of $\mathcal{L}_{\text {LDAA }}$ via the following translation function $t r: \mathcal{L}_{\mathrm{LPA}} \longrightarrow \mathcal{L}_{\mathrm{LDAA}}$ which is defined as follows:

$-\operatorname{tr}(p)=p$ for $p \in$ Atm

$-\operatorname{tr}(\neg \varphi)=\neg \operatorname{tr}(\varphi)$

$-\operatorname{tr}\left(\varphi_{1} \wedge \varphi_{2}\right)=\operatorname{tr}\left(\varphi_{1}\right) \wedge \operatorname{tr}\left(\varphi_{2}\right)$

$-\operatorname{tr}\left(A_{i} \varphi\right)=\bigcirc_{i} \operatorname{tr}(\varphi)$

$-\operatorname{tr}\left(B_{i} \varphi\right)=\square_{i} \operatorname{tr}(\varphi)$

$-\operatorname{tr}\left(X_{i} \varphi\right)=\bigcirc_{i} \operatorname{tr}(\varphi) \wedge \square_{i} \operatorname{tr}(\varphi)$

The interesting aspect of the previous translation is that the LPA notion of explicit belief is mapped into the combination of implicit belief plus awareness in our logic LDAA, and not directly into the LDAA notion of explicit belief. This highlights that the two notions of explicit belief do not capture the same type of epistemic attitude. While the LDAA notion represents an agent's actual belief which is active and accessible in his working memory (we assume an agent's belief base to be a rough approximation of his working memory), the LPA notion is aimed at capturing the agent's beliefs that are built from his vocabulary and therefore understandable by him. ${ }^{4}$

As the following theorem highlights, the translation is satisfiability preserving.

Theorem 3. Let $\varphi \in \mathcal{L}_{\mathrm{LPA}}$. Then, $\varphi$ is satisfiable for the class PAM if and only if $\operatorname{tr}(\varphi)$ is satisfiable for the class NDAM.

Proof. We first prove a weaker result of the left-to-right direction, i.e., if $\varphi$ is satisfiable for the class PAM, then $\operatorname{tr}(\varphi)$ is satisfiable for the class QNDAM. Let $\mathcal{M}=(\mathrm{S}, \mathrm{R}, \rho, \pi)$ be a PAM and let $s \in \mathrm{S}$ such that $(\mathcal{M}, s) \models \varphi$. We build the corresponding $\mathcal{M}^{\prime}=(\mathrm{W}, \mathrm{D}, \mathrm{A}, \mathrm{N}, \mathrm{V})$ as follows:

$-\mathrm{W}=\mathrm{S}$,

\footnotetext{
${ }^{4}$ Note that if we defined the translation sending explicit beliefs of LPA into explicit beliefs of LDAA, satisfiability would be preserved only in the direction from LDAA to LPA. For the other direction, a formula of the form $X_{i} B_{i} \varphi$ in $\mathcal{L}_{\mathrm{LPA}}$ cannot be translated into $\mathcal{L}_{\text {LDAA }}$ with this alternative translation.
} 
- $\forall i \in$ Agt and $\forall s \in \mathrm{S}, \mathrm{D}(i, s)=\left\{p \vee \neg p \mid(\mathcal{M}, s) \models A_{i} p\right\}$,

- $\forall i \in$ Agt and $\forall s \in \mathrm{S}, \mathrm{A}(i, s)=\rho(i, s)$,

$-\forall i \in A g t$ and $\forall s \in \mathrm{S}, \mathrm{N}(i, s)=\mathrm{R}(i, s)$,

$-\forall p \in \operatorname{Atm}, \mathrm{V}(p)=\pi(p)$.

We prove that $\mathcal{M}^{\prime}$ is a quasi-NDAM by showing that it satisfies Condition $\left(\mathrm{C} 1^{*}\right)$ and $\left(\mathrm{C} 2^{*}\right)$ in Definition 12.

For Condition $(\mathrm{C} 1 *)$, by the semantics of PAM and the definitions of $\mathrm{D}(i, s)$ and $\mathrm{A}(i, s)$, it is easy to show that, $\operatorname{Atm}(\mathrm{D}(i, s))=\mathrm{A}(i, s)$, which implies that, $\operatorname{Atm}(\mathrm{D}(i, s)) \subseteq \mathrm{A}(i, s)$.

For Condition $\left(\mathrm{C} 2^{*}\right)$, by the definition of $\mathrm{D}(i, s)$, there are only tautologies in it. So we have that $\bigcap_{\operatorname{tr}(\varphi) \in \mathrm{D}(i, w)}\|\operatorname{tr}(\varphi)\|_{M}=\mathrm{W}$. Then, clearly, Condition $\left(\mathrm{C} 2^{*}\right)$ is satisfied.

It is easy to verify that, for every $x \in\{\mathrm{GC}, \mathrm{BC}\}$, if $\mathcal{M}$ satisfies $x$ then $\mathcal{M}^{\prime}$ satisfies it as well.

By induction on the structure of $\varphi$, we prove that, $\forall s \in \mathrm{S},(\mathcal{M}, s) \models \varphi$ iff $\left(\mathcal{M}^{\prime}, s\right) \models \operatorname{tr}(\varphi)$.

For the case $\varphi=p$ and the boolean cases $\varphi=\neg \psi$ and $\varphi=\psi_{1} \wedge \psi_{2}$, it is straightforward.

Now we consider the case $\varphi=A_{i} \psi$. Suppose $(\mathcal{M}, s) \models A_{i} \psi$. By the semantics of PAM, it is equivalent to $\operatorname{Atm}(\psi) \subseteq \rho(i, s)$. By the definition of $\mathrm{A}(i, s)$ and the function $\operatorname{tr}$, the latter is equivalent to $\operatorname{Atm}(\operatorname{tr}(\psi)) \subseteq \mathrm{A}(i, s)$. And in turn the latter means $\left(\mathcal{M}^{\prime}, s\right) \models \bigcirc_{i} \operatorname{tr}(\psi)$. Then, by the definition of the function $t r$, the latter is equivalent to $\left(\mathcal{M}^{\prime}, s\right) \models \operatorname{tr}\left(A_{i} \psi\right)$.

Let us consider the case $\varphi=B_{i} \psi$. Suppose $(\mathcal{M}, s) \models B_{i} \psi$. By the induction hypothesis, we have $\|\psi\|_{\mathcal{M}}=\|\operatorname{tr}(\psi)\|_{\mathcal{M}^{\prime}} .(\mathcal{M}, s) \models B_{i} \psi$ means that $\mathrm{R}(i, s) \subseteq$ $\|\psi\|_{\mathcal{M}}$. By the definition of $\mathrm{N}(i, s)$ and the fact that $\|\psi\|_{\mathcal{M}}=\|\operatorname{tr}(\psi)\|_{\mathcal{M}^{\prime}}$, the latter it equivalent to $\mathrm{N}(i, s) \subseteq\|\operatorname{tr}(\psi)\|_{\mathcal{M}^{\prime}}$, which is equivalent to $\left(\mathcal{M}^{\prime}, s\right) \models$ $\square_{i} \operatorname{tr}(\psi)$. The latter means $\left(\mathcal{M}^{\prime}, s\right) \models \operatorname{tr}\left(B_{i} \psi\right)$ by the definition of the function tr.

Finally, let us consider the case $\varphi=X_{i} \psi$. Suppose $(\mathcal{M}, s) \models X_{i} \psi$. Given the fact that $X_{i} \psi$ is equivalent to $B_{i} \psi \wedge A_{i} \psi$, by the previous cases, it means that, $\left(\mathcal{M}^{\prime}, s\right) \models \square_{i} \operatorname{tr}(\psi) \wedge \bigcirc_{i} \operatorname{tr}(\psi)$. By the function $\operatorname{tr}$, the latter is equivalent to $\left(\mathcal{M}^{\prime}, s\right) \models \operatorname{tr} X_{i} \psi$.

Thus, we conclude that $(\mathcal{M}, s) \models \varphi$ iff $\left(\mathcal{M}^{\prime}, s\right) \models \operatorname{tr}(\varphi)$ for all $s \in \mathrm{S}$. Then we have that, if $\varphi$ is satisfiable for the class $\mathbf{P A M}$, then $\operatorname{tr}(\varphi)$ is satisfiable for the class QNDAM. By Theorem 1, it follows that, if $\varphi$ is satisfiable for the class PAM, then $\operatorname{tr}(\varphi)$ is satisfiable for the class NDAM.

Then we prove the right-to-left direction. Let $\mathcal{M}=(\mathrm{W}, \mathrm{D}, \mathrm{A}, \mathrm{N}, \mathrm{V})$ be a NDAM. We build the model $\mathcal{M}^{\prime}=(\mathrm{S}, \mathrm{R}, \rho, \pi)$ as follows:

$-\mathrm{S}=\mathrm{W}$

- $\forall i \in$ Agt and $\forall w \in \mathrm{W}, \mathrm{R}(i, w)=\mathrm{N}(i, w)$,

- $\forall i \in A g t$ and $\forall w \in \mathbf{W}, \rho(i, w)=\mathbf{A}(i, w)$,

$-\forall p \in \operatorname{Atm}, \pi(p)=\mathrm{V}(p)$. 
It is easy to show that $\mathcal{M}^{\prime}$ is a PAM.

The next step is to prove that for all $w \in \mathrm{W},(\mathcal{M}, w) \models \operatorname{tr}(\varphi)$ iff $\left(\mathcal{M}^{\prime}, w\right) \models \varphi$.

The case $\varphi=p$ and the boolean cases are straightforward.

Let us consider the case $\varphi=A_{i} \psi$. Suppose $(\mathcal{M}, w) \models \operatorname{tr}\left(A_{i} \psi\right)$. By the semantics of NDAM and the function $t r$, it is equivalent to $\operatorname{Atm}(\psi) \subseteq \mathrm{A}(i, w)$. By the definition of $\rho(i, w)$, the latter is equivalent to $\operatorname{Atm}(\psi) \subseteq \rho(i, w)$. Then by the semantics of PAM, the latter is equivalent to $\left(\mathcal{M}^{\prime}, w\right) \models A_{i} \psi$.

Let us consider the case $\varphi=B_{i} \psi$. Suppose $(\mathcal{M}, w) \models \operatorname{tr}\left(B_{i} \psi\right)$. By the induction hypothesis, we have $\|\psi\|_{\mathcal{M}^{\prime}}=\|\operatorname{tr}(\psi)\|_{\mathcal{M}}$. By the function $\operatorname{tr},(\mathcal{M}, w) \mid=$ $\operatorname{tr}\left(B_{i} \psi\right)$ means $(\mathcal{M}, w) \models \square_{i} \operatorname{tr}(\psi)$. By the semantics of NDAM, the latter is equivalent to $\mathrm{N}(i, w) \subseteq\|\operatorname{tr}(\psi)\|_{\mathcal{M}}$. By the definition of $\mathrm{R}(i, w)$ and the fact $\|\psi\|_{\mathcal{M}^{\prime}}=\|\operatorname{tr}(\psi)\|_{\mathcal{M}}$, the latter is equivalent to $\mathrm{R}(i, w) \subseteq\|\psi\|_{\mathcal{M}^{\prime}}$, which is equivalent to $\left(\mathcal{M}^{\prime}, w\right) \models B_{i} \psi$

Finally, let us consider the case $\varphi=X_{i} \psi$. Suppose $(\mathcal{M}, w) \models \operatorname{tr}\left(X_{i} \psi\right)$. Given the the fact that $X_{i} \psi$ is equivalent to $B_{i} \psi \wedge A_{i} \psi$, by the previous cases, it is equivalent to $\left(\mathcal{M}^{\prime}, w\right) \models B_{i} \psi \wedge A_{i} \psi$, which in turn is equivalent to $\left(\mathcal{M}^{\prime}, w\right) \models$ $X_{i} \psi$.

Thus, we conclude that $(\mathcal{M}, w) \models \operatorname{tr}(\varphi)$ iff $\left(\mathcal{M}^{\prime}, w\right) \models \varphi$ for all $w \in \mathrm{W}$. Then we have that, if $\operatorname{tr}(\varphi)$ is satisfiable for the class NDAM, then $\varphi$ is satisfiable for the class PAM.

Theorem 3 shows that the translation of any satisfiable LPA-formula is satisfiable relative to NDAM models. This highlights that $\mathcal{L}_{\text {LDAA }}$ is at least as expressive as the translated version $\mathcal{L}_{\text {LPA }}$ with repect to the class NDAM. We do not know whether the the other direction works as well. What we can affirm is that the formula $\neg \triangle_{i}(p \wedge p) \wedge \square_{i} p \wedge \bigcirc_{i} p$ is satisfiable in the class NDAM, but it cannot be satisfied in the class PAM, if we translated $\triangle_{i}, \square_{i}$, and $\bigcirc_{i}$ into $X_{i}$, $B_{i}$, and $A_{i}$, respectively. Again this shows that the LPA notion of explicit belief and the LDAA notion of explicit belief capture epistemic attitudes of different nature.

\section{Conclusion}

We have provided a novel investigation of propositional awareness and of its relationship with explicit and implicit belief. In our approach, explicit belief is the only primitive concept, and awareness and implicit belief are grounded on it. Specifically, an agent's awareness set and set of doxastic alternatives are directly computed from the agent's belief base. The main results of the paper are an axiomatics for our logic of awareness, explicit and implicit belief as well as a polynomial embedding of Halpern's logic of propositional awareness into our logic. Future work will be devoted to explore more properties of awareness typically discussed in the literature, such as beliefs of awareness and unawareness (also known as awareness/unawareness introspection), and the dynamic aspects of awareness and beliefs. We expect our approach to offer a new foundation for the dynamics of awareness, alternative to [2], in which awareness change is anchored in belief base change. 


\section{Acknowledgements}

This work is supported by the major project of Key Research Institutes of Humanities and Social Sciences of Ministry of Education of China (No. 17JJD720008), and by the key project of National Social Science Foundation of China (No. 16AZX017). Support from the ANR-3IA Artificial and Natural Intelligence Toulouse Institute (ANITI) is also gratefully acknowledged.

\section{References}

1. Alchourrón, C.E., Gärdenfors, P., Makinson, D.: On the logic of theory change: partial meet contraction and revision functions. Journal of Symbolic Logic. 50(2), 510-530 (1985)

2. van Benthem, J., Velázquez-Quesada, F. R.: The dynamics of awareness. Synthese, 177, 5-27 (2010)

3. van Ditmarsch, H., French, T., Velázquez-Quesada, F., Wang, Y.: Implicit, explicit and speculative knowledge. Artificial Intelligence, 256, 35-67 (2018)

4. Fagin, R., Halpern, J.: Belief, awareness, and limited reasoning. Artificial Intelligence, 34(1), 39-76 (1988)

5. Halpern, J.: Alternative semantics for unawareness. Games and Economic Behavior, 37(2), 321-339 (2001)

6. Halpern, J., Rêgo, L.: Interactive unawareness revisited. Games and Economic Behavior, 62(1), 232-262 (2008)

7. Hansson, S. O.: Theory contraction and base contraction unified. Journal of Symbolic Logic, 58(2), 602-625 (1993)

8. Heifetz, A., Meier, M., Schipper, B.: Interactive unawareness. Journal of Economic Theory, 130, 78-94 (2006)

9. Heifetz, A., Meier, M., Schipper, B.: A canonical model for interactive unawareness. Games and Economic Behavior, 62, 304-324 (2008)

10. Hintikka, J.: Knowledge and belief. Cornell University Press, Ithaca (1962)

11. Levesque, H.: A logic of implicit and explicit belief. In: Proceedings of AAAI 1984, pp. 198-220. AAAI Press (1984)

12. Lorini, E.: Rethinking Epistemic Logic with Belief Bases. Artificial Intelligence, 282 (2020)

13. Lorini, E.: In Praise of Belief Bases: Doing Epistemic Logic Without Possible Worlds. In: Proceedings of AAAI 2018, pp. 1915-1922. AAAI Press (2018)

14. Makinson, D.: How to give it up: A survey of some formal aspects of the logic of theory change. Synthese, 62, 347-363 (1985)

15. Modica, S., Rustichini, A.: Awareness and partitional information structures. Theory and Decision, 37, 107-124 (1994)

16. Modica, S., Rustichini, A.: Unawareness and partitional information structures. Games and Economic Behavior, 27, 265-298 (1999)

17. Nebel, B.: Syntax-based approaches to belief revision. In: Gärdenfors, P. (eds.) Belief Revision, pp. 52-88. Cambridge University Press (1992)

18. Rott, A.: "Just because": Taking belief bases seriously. In: Logic Colloquium 98: Proceedings of the 1998 ASL European Summer Meeting. In: Lecture Notes in Logic, vol. 13, pp. 387-408. Association for Symbolic Logic (1998)

19. Stalnaker, R.: Common Ground. Linguistics and Philosophy, 25(5-6), 701-721 (2002) 